\title{
Study of level structure on ${ }^{26} \mathrm{Si}$ for the astrophysical interest in ${ }^{26} \mathrm{Al}$ production
}

\author{
Tetsuro Komatsubara ${ }^{1}$, Akira Ozawa ${ }^{1}$, Tetsuaki Moriguchi ${ }^{4}$, Yuta Ito ${ }^{1}$, Yoko \\ Ishibashi ${ }^{1}$, Yasushi Abe ${ }^{1}$, Toshiaki Yuasa ${ }^{1}$, Takehito Hayakawa ${ }^{2}$, Toshiyuki \\ Shizuma ${ }^{2}$, Kaoru Y. Hara ${ }^{2}$, Shigeru Kubono ${ }^{3}$, Hidetoshi Yamaguchi ${ }^{5}$, Daid Kahl ${ }^{5}$, \\ Seiya Hayakawa ${ }^{6}$, Dam N. Binh ${ }^{7}$, Alan A. Chen ${ }^{8}$, Jun Chen ${ }^{8}, K_{i a n a}$ Setoodehnia ${ }^{8}$, \\ Takahiro Kajino ${ }^{9}$ \\ ${ }^{1}$ Division of Physics, University of Tsukuba \\ Tennodai 1-1-1, Tsukuba, Ibaraki 305-8577, Japan \\ E-mail: komatsubara@tac. tsukuba.ac.jp \\ ${ }^{2}$ Japan Atomic Energy Agency, \\ Shirakata Shirane 2-4, Tokai, Ibaraki 319-1195, Japan \\ ${ }^{3}$ Riken, Hirosawa 2-1, Wako, Saitama 351-0198, Japan \\ ${ }^{4}$ National Cerebral and Cardiovascular Center, Fujishirodai, Suita, Osaka 565-8565, Japan \\ ${ }^{5}$ Center for Nuclear Study (CNS), the University of Tokyo, Hirosawa 2-1, Wako, Saitama 351-0198, \\ Japan \\ ${ }^{6}$ Dipartimento di Fisica e Astronomia, Universita di Catania, Catania, Italy \\ ${ }^{7}$ Institute of Physics, Vietnamese Academy for Science and Technology, Vietnam \\ ${ }^{8}$ Department of Physics \& Astronomy, McMaster University, Hamilton, ON L8S 4M1, Canada \\ ${ }^{9}$ National Astronomical Observatory, Osawa, Mitaka, Tokyo 181-8588, Japan
}

A radio-isotope of ${ }^{26 \mathrm{~g}} \mathrm{Al}$ (where $\mathrm{g}$ is expressing the ground state) is known as a $\gamma$-ray emitter spreading in the Milky Way galaxy. Since the half-life is only $7.2 \times 10^{5}$ year this is the obvious evidence of ongoing nucleosynthesis in our galaxy. This isotope is also known as an origin of abundance-anomaly of ${ }^{26} \mathrm{Mg}$ observed in meteorites. Therefore, investigations of production rates for the ${ }^{26 \mathrm{~g}} \mathrm{Al}$ are quite important for the nucleosynthesis occurring in massive star, classical nova and super nova. Although, there is still some uncertainties in the nuclear structure of ${ }^{26} \mathrm{Si}$ which is located at the bypass sequence producing the isomeric state ${ }^{26 \mathrm{~m}} \mathrm{Al}$ (where $\mathrm{m}$ is expressing the metastable $1^{\text {st }}$ excited state). For the comprehensive understanding of the nuclear reaction rate to produce the ${ }^{26 \mathrm{~g}} \mathrm{Al}$, we have done $\gamma$-ray spectroscopy to investigate the level structure for the ${ }^{26} \mathrm{Si}$. We confirmed the presence of a recently proposed level located just above the proton threshold. Furthermore, as a result of the gamma-ray angular correlation measurements we could assign the spin of this level as $0^{+}$.

XII International Symposium on Nuclei in the Cosmos

August 5-12, 2012

Cairns, Australia

1 Speaker: Tetsuro Komatsubara 


\section{Introduction}

Recent observations of $1.809 \mathrm{MeV} \gamma$-rays by balloon and satellite observatories[1-3] revealed that the $\gamma$-ray emitters of ${ }^{26 g} \mathrm{Al}$ are spreading over the Milky Way and rotating with its motion[3]. Since the half-life of the ${ }^{26 \mathrm{~g}} \mathrm{Al}$ is only $7.2 \times 10^{5}$ year, it surely proves that the nucleosynthesis is still proceeding in our galaxy. Origin of the ${ }^{26 \mathrm{~g}} \mathrm{Al}$ could be considered to be massive stars like as Wolf Rayet stars and/or super novae. Classical novae could also have some contribution of the abundance[4]. This isotope is also known as an origin of abundance-anomaly of ${ }^{26} \mathrm{Mg}$ found in meteorites[5]. The theoretical calculation to reproduce the abundance of the ${ }^{26 \mathrm{~g}} \mathrm{Al}$ is quite important for the study of nucleosynthesis. However, there are still uncertainties of the reaction rates specially concerning about excited structure of ${ }^{26} \mathrm{Si}$ which is located at a bypass sequence to produce the isomeric state ${ }^{26 \mathrm{~m}} \mathrm{Al}[6]$ as shown in Fig. 1. Once the ${ }^{26} \mathrm{Si}$ decays into the ${ }^{26 \mathrm{~m}} \mathrm{Al}$ this state directly feeds into the ground state of ${ }^{26} \mathrm{Mg}$ without the $1.809 \mathrm{MeV}$ gamma ray emission. For the comprehensive knowledge of the production of the ${ }^{26 \mathrm{~g}} \mathrm{Al}$, we have done $\gamma$-ray spectroscopy to investigate the level structure of ${ }^{26} \mathrm{Si}$.

Creation of the ${ }^{26 g} \mathrm{Al}$ would be proceeding by a reaction of ${ }^{25} \mathrm{Al}\left(\mathrm{e}^{+} v\right)^{25} \mathrm{Mg}(p, \gamma){ }^{26 \mathrm{~g}} \mathrm{Al}$. Whereas, if the circumstance is high temperature like as super novae or classical novae, another bypass sequence opens to be ${ }^{25} \mathrm{Al}(p, \gamma)^{26} \mathrm{Si}\left(e^{+} v\right)^{26 \mathrm{~m}} \mathrm{Al}\left(e^{+} v\right)^{26} \mathrm{Mg}$ where the reaction flow reaches to the isomeric (metastable) state $0^{+}\left(\mathrm{T}_{1 / 2}=6.3 \mathrm{sec}\right)$ of ${ }^{26 \mathrm{~m}} \mathrm{Al}$ and feeds directly into the ground state of ${ }^{26} \mathrm{Mg}$. Therefore, if the ${ }^{26} \mathrm{Si}$ is created the $1.809-\mathrm{MeV} \gamma$-ray cannot be emitted as shown in Fig. 1.

The reaction flow of ${ }^{25} \mathrm{Al}(p, \gamma)^{26} \mathrm{Si}$ is considered as admixture of a direct process and resonant captures[6]. Because spins of ${ }^{25} \mathrm{Al}$ and proton are $5 / 2^{+}$and $1 / 2^{+}$, respectively, the predominant flow might proceed through $3^{+}$just above the proton threshold in ${ }^{26} \mathrm{Si}[6,7]$. There are many efforts to seek the states by using neutron TOF[8], light ion reaction studies[9-14], $\beta$-delayed proton emission spectroscopy[16] and $\gamma$-ray spectroscopies[16]. Indeed, extensive studies with radioactive ion beams have been reported[17-19]. Furthermore, there are several brief reports proposing a new level around $E_{x}=5.888$ $\mathrm{MeV}[20,21]$. In order to investigate the level structure of the ${ }^{26} \mathrm{Si}$ we have carried out inbeam $\gamma$-ray spectroscopy at University of Tsukuba.

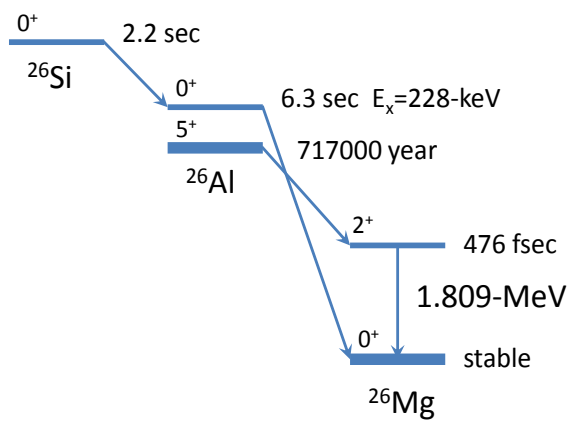

Fig. 1. A sequence through ${ }^{26} \mathrm{Si}$ feeding into a $6.3 \mathrm{sec}$ isomeric state ${ }^{26 \mathrm{~m}} \mathrm{Al}$ where the $1.809-\mathrm{MeV} \gamma$ ray cannot be emitted. Decay of the ground state ${ }^{26 g} \mathrm{Al}$ is also shown with the $1.809-\mathrm{MeV} \gamma$-ray emission. 


\section{Experimental procedure}

Present in-beam $\gamma$-ray spectroscopy experiments include $\gamma$-ray singles, angular distribution, $\gamma-\gamma$ and $\gamma-\gamma-n$ coincidences which are performed using the ${ }^{24} \mathrm{Mg}\left({ }^{3} \mathrm{He}, n\right){ }^{26} \mathrm{Si}$ reaction at the University of Tsukuba Tandem Accelerator Complex (UTTAC). A ${ }^{3} \mathrm{He}$ beam of $10 \mathrm{MeV}$ was irradiated on a natural magnesium target, where the abundance of the ${ }^{24} \mathrm{Mg}$ is $78.99 \%$. As the nuclear reactions between the magnesium and ${ }^{3} \mathrm{He}$, many other reaction channels opened into ${ }^{23} \mathrm{Mg},{ }^{24} \mathrm{Mg},{ }^{25} \mathrm{Mg},{ }^{26} \mathrm{Mg},{ }^{25} \mathrm{Al},{ }^{26} \mathrm{Al}$, and ${ }^{28} \mathrm{Si}$. Probability to produce the ${ }^{26} \mathrm{Si}$ was estimated to be only $1 \%$ of total fusion cross section. However the measurements of $\gamma-\gamma$ and $\gamma-\gamma-n$ coincidences can clearly identify transitions of the ${ }^{26} \mathrm{Si}$ by using high resolution germanium detectors.

For the measurements of $\gamma$-rays, because the most important $\gamma$-transition is expected around 4-MeV, we used rather large high purity germanium (HPGe) detectors whose efficiencies are $140 \%, 70 \%$ and $50 \%$ relative to the efficiency of 3 -inch $\times 3$-inch NaI detector. For the neutron detection we used two liquid scintillation detectors located at nearly zero degree with respect to the beam axis. The angular distribution measurement of $\gamma$-ray singles has been carried out at the angles of $90^{\circ}, 100^{\circ}, 110^{\circ}, 120^{\circ}, 130^{\circ}$, and $140^{\circ}$ for the $1797.5-\mathrm{keV}$ transition as described in the following sub-section. The angular correlation measurement has been performed with two Ge detectors which were located at either $\left(\theta_{1}, \varphi_{1}, \theta_{2}, \varphi_{2}\right)=\left(90^{\circ}, 0^{\circ}, 90^{\circ}, 180^{\circ}\right)$ or $\left(90^{\circ}, 0^{\circ}, 135^{\circ}, 180^{\circ}\right)$. Ratios of the measured intensities defined as $r=I\left(90^{\circ}, 0^{\circ}, 135^{\circ}, 180^{\circ}\right) / I\left(90^{\circ}, 0^{\circ}, 90^{\circ}, 180^{\circ}\right)$ called as DCO ratios were evaluated and compared with theoretical calculations[22].

The energies and efficiencies of the detectors were calibrated using standard radioactive sources of ${ }^{56} \mathrm{Co}$ and ${ }^{152} \mathrm{Eu}$. Furthermore, $\gamma$-rays emitted from ${ }^{25} \mathrm{Mg},{ }^{26} \mathrm{Mg}$, and ${ }^{26} \mathrm{Al}$ which are the by-products from the natural magnesium $+{ }^{3} \mathrm{He}$ reactions were also used for the energy calibration. The systematical uncertainty for $\gamma$-ray energy is estimated to be $0.5 \mathrm{keV}$ for the energy region between 400 and $3200 \mathrm{keV}$. This is also estimated to be $0.8 \mathrm{keV}$ around $4000 \mathrm{keV}$ where the estimation includes additional uncertainties derived from the quadrant term applying outside of the calibration points[22].

\section{Levels structure}

In-beam $\gamma$-ray spectroscopic studies for ${ }^{26} \mathrm{Si}$ were reported by Rolfs[23], Bell[24], and recently by Seweryniak[16] with GAMMASPHERE. Furthermore there are several brief reports [20,21] proposing a new level around 5888-keV which would be one of the most important levels for the resonant reaction rate to create ${ }^{26} \mathrm{Si}$. We confirmed all of levels observed by Seweryniak[16]. However, levels at 3842- and 4093- keV reported by Rolfs and at 3820-keV by Bell could not be assigned. Those levels were neither reported by Seweryniak[16]. Moreover, we confirmed the 4091.1-keV transition, which Rofls firstly observed and attributed to the 4093-keV level. This corrected placement agrees with the recent brief reports[20,21]. The spin of the 5888.6-keV level was assigned as $0^{+}$from the $\gamma-\gamma$ angular correlation measurement[22].

\subsection{5-keV level}

The $1797.5-\mathrm{keV}$ level is the $1^{\text {st }}$ excited state of ${ }^{26} \mathrm{Si}$ that has a spin of $2^{+}$and it emits $1797.5-\mathrm{keV}$ transition which is well observed in our neutron coincidence spectrum. This 
$\gamma$-ray is only one belonged to ${ }^{26} \mathrm{Si}$ which can be clearly observed in the singles measurements. Then, $\gamma$-ray angular distribution measurement has been carried out for this $1797.5-\mathrm{keV}$ transition. However, the singles spectrum measured at large angles around $140^{\circ}$ shows serious Doppler broadening shape[22]. Furthermore, the measured result of the $\gamma$-ray angular distribution was strongly distorted and quite different from typical stretched E2 transition of $2 \rightarrow 0$ [22]. Unknown strong perturbation might occur inside of the magnesium target to distort the angular distribution. For this reason, rather large value of $\sigma / \mathrm{J}=2$ is adapted for the initial population of magnetic sub-state in the analysis of the $\gamma-\gamma$ angular correlation[22].

\subsection{8-keV level}

This level is the second excited state of ${ }^{26} \mathrm{Si}$ having spin of $2^{+}$and feeding into both the $1^{\text {st }}$ and the ground states emitting 989.1- and 2786.9-keV transitions, respectively. The 989.1-keV $\gamma$-ray has been clearly seen in the neutron coincidence spectrum. This $\gamma$-ray has also been observed in the singles spectra, however, the peak is known to make a doublet with $989.9-\mathrm{keV}$ transition of ${ }^{25} \mathrm{Mg}$. Then the angular distribution of the singles measurement couldn't have proper information for the spin assignment.

The 989.1-keV transition has the second strongest intensity in all of $\gamma$-rays of ${ }^{26} \mathrm{Si}$, so that the gated spectrum by this $\gamma$-ray is clean and informative. This transition is known as M1/E2 mixed transition having the mixing ratio $\delta=-0.21 \pm 0.10$ by Rolfs[23] in their neutron coincident angular distribution measurement. In our analysis of the angular correlation measurements, this value is adapted as the calibration[22].

\subsection{6-keV level}

This level is known as $0^{+}$state feeding into the $1^{\text {st }}$ excited state through $1538.1-\mathrm{keV}$ transition. The intensity of this transition is the third strongest in our $\gamma$-ray intensity table which is $32.2 \%$ of the strongest $1797.5-\mathrm{keV}$ transition. Seweryniak[16] reported that the intensity of this transition is only $2.6 \%$ of the $1797.5-\mathrm{keV}$ transition. This difference could be due to the entrance-angular-momentum-window of fusion reactions. Seweryniak used the ${ }^{12} \mathrm{C}\left({ }^{16} \mathrm{O}, 2 n\right)^{26} \mathrm{Si}$ reaction. The maximum angular momenta can be estimated to be 5.0 and $16.2 \mathrm{~h}$ for the present study and Seweryniak's, respectively.

\subsection{9-keV level}

This level emits 971.1- and 1960.4-keV transitions and agrees with the reports by Bell[24] and Seweryniak[16]. Measured ratios of angular correlation for the 1960.4$1797.5-\mathrm{keV}$ cascade is consistent to the $3^{+}$assignment[22].

\subsection{1-keV level}

This level emits 1351.9- and 2341.6-keV transitions and is consistent to reports done by Rolfs[23], Bell[24] and Seweryniak[16]. They also reported one more transition having an energy of 4441-keV connecting to the ground state.

\subsection{1-, 4445.4-, and 4795.1-keV level}

The 4186.1-keV level emits 1400.5- and 2387.4-keV transitions. This location is consistent to the Seweryniak's report[16]. The 4445.4-keV level is observed by emitting 
2647.9-keV transition connecting to the $1^{\text {st }}$ excited state. This level was firstly reported by Bell[24]. Severyniak observed other transition having $1660-\mathrm{keV}$ fed into $2786-\mathrm{keV}$ level which cannot be observed in our results. The 4795.1-keV level is observed to feed into the $1^{\text {st }}$ excited state through $2997.6-\mathrm{keV} \gamma$-ray which has firstly been reported by Seweryniak[16].

\subsection{7- and 4830.6-keV levels}

The 4808.7- and 4830.6-keV levels are decaying into the $2^{\text {nd }}$ excited state by emitting 2012.9- and 2043.8-keV $\gamma$-rays, respectively. Spin assignments of both levels were only tentatively given by Seweryniak from the discussion of the intensity balance. We have confirmed these spin assignments by our angular correlation results[22] to be $2^{+}$and $0^{+}$, respectively.

\subsection{0-, 5286.8, and 5517.2-keV level}

These levels were firstly reported by Seweryniak[16]. We observed almost the same cascade relations. However, a few transitions in his report[16] cannot be observed in our coincidence matrix.

\subsection{3-keV level}

This level is one of the most important levels for the nucleosynthesis of ${ }^{26} \mathrm{Si}$ which are just located above the proton threshold. The level was firstly reported by Parpottas with his neutron TOF spectra. He assigned its spin as $1^{+}$comparing its cross-section with Hauser-Feshbach calculation. Seweryniak[16] has observed the 3879-keV transition by using GAMMASPHERE detector array combined with a recoil mass analyzer. However, no information of either $\gamma$-ray angular distribution or angular correlation has been reported. We observed $3874.8-\mathrm{keV}$ transition which agrees with the previous reports. Our result of the angular correlation can support its $1^{+}$spin assignment[22].

\subsection{6-keV level}

As the result of the present study, the newly proposed level[20,21] at 5888.6-keV has been confirmed. This level is emitting 3101.6- and 4091.1-keV $\gamma$-rays feeding into the $1^{\text {st }}$ and the ground states, respectively. On the other hand the 4095-keV gamma ray was observed to belong to ${ }^{26} \mathrm{Si}$ by Bell[24], he allocated it to the different level at $4095-\mathrm{keV}$ which cannot be observed in our result. Then, we verified the previous reports[20,21]. However, there is a discrepancy that de Séréville[21] reported a rather strong $\gamma$-transition having an energy of 1750$\mathrm{keV}$ decaying from this level to the $4139-\mathrm{keV}$ level in his neutron coincident $\gamma$-ray spectrum.

Spin assignment of this level was performed by angular correlation measurements. We observed that the ratio for the 4091.1 - 1797.5-keV cascade is quit similar to those for 1538.1 $1797.5-\mathrm{keV}$ cascade which is known as $0 \rightarrow 2 \rightarrow 0$ cascade. A calculated value with consideration of finite solid angle of detectors also agrees with this observation. Then, we confirmed spin of the level is $0^{+}$.

By comparison with shell model calculation and states of mirror nucleus ${ }^{26} \mathrm{Mg}$, there could be three levels just above the proton threshold which are $0^{+}, 1^{+}$and $3^{+}[6]$. Parpottas[7] reported a state at $5946-\mathrm{keV}$ as rather faint peak in his neutron TOF spectrum and assigned as $0^{+}$from the comparison with Hauser-Feshbach calculation. However, there is no more report to support his observation, except Caggiano's report done by $\left({ }^{3} \mathrm{He},{ }^{6} \mathrm{He}\right)$ reaction[8]. 


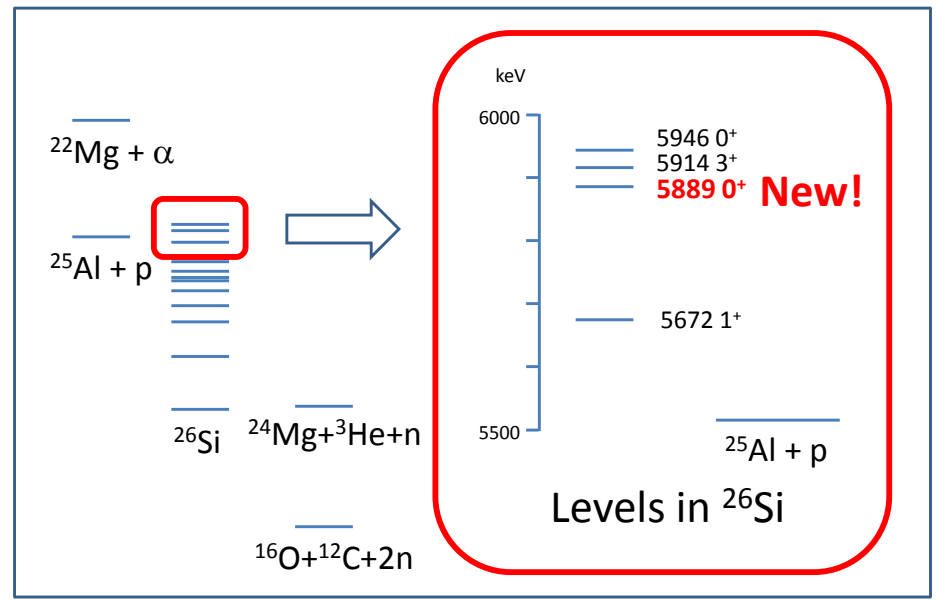

Fig. 2 Levels just above the proton threshold in ${ }^{26} \mathrm{Si}$.

\section{Summary}

For the study of nucleosynthesis of ${ }^{26 \mathrm{~g}} \mathrm{Al}$, level structure of ${ }^{26} \mathrm{Si}$ has been investigated via in-beam $\gamma$-ray spectroscopy. Existence of the newly proposed level[20,21] at 5888.6 $\pm 0.9-\mathrm{keV}$ has been confirmed. Spin parity of this level has been newly determined as $0^{+}$ by the angular correlation measurements[22].

This work has been supported in part by Grants-in-Aid for Scientific Reaches (21340068 and 21540295) of Japan.

\section{References}

[1] W.A. Mahoney, et al., The Astrophysical Journal 286 (1984) 578.

[2] R. Diehl, et al., Astron. Astrophys. 298 (1995) 445.

[3] R. Diehl, et al., Nature 439 (2006) 45.

[4] J. Jose, et al., Astrop. J. 520 (1999) 347.

[5] T. Lee, et al., Astrop. J. 211 (1977) L107.

[6] C. Iliadis, et al., Phys. Rev. C 53 (1996) 475.

[7] T. Eronen, et al., Phys. Rev. C 79 (2009) 032802(R).

[8] Y. Parpottas, et al., Phys. Rev. C 70 (2004) 065805.

[9] J.A. Caggiano, et al., Phys. Rev. C 65 (2002) 055801.

[10] D.W. Bardayan, et al., Phys. Rev. C 65 (2002) 032801(R).

[11] D.W. Bardayan, et al., Phys. Rev. C 74 (2006) 045804.

[12] A. Matic, et al., Phys. Rev. C 82 (2010) 025807.

[13] K. A. Chipps, et al. Phys. Rev. C 82 (2010) 045803.

[14] Y.K. Kwon, et al., Journal of the Korean Physical Society 53 (2008) 1141.

[15] J.C. Thomas, et al., Eur. Phys. J A 21 (2004) 419.

[16] D. Seweryniak, et al., Phys. Rev. C 75 (2007) 062801(R).

[17] P.N. Peplowski, et al., Phys. Rev. C 79 (2009) 032801(R).

[18] J. Chen, et al., Phys. Rev. Nucl. Phys. A 834 (2010) 667c.

[19] J. Chen, et al., Phys. Rev. C 85 (2012) 015805.

[20] T. Komatsubara, et al., UTTAC Annual Report 2007, UTTAC-77 (2008) 15.

[21] N. de Séréville, et al. in Proceedings of the $11^{\text {th }}$ Symposium on Nuclei in the Cosmos, NIC XI,

July 19-23, (2010), PoS(NïC XI) 212.

[22] T. Komatsubara, et al., to be published.

[23] C. Rolfs and W. Trost, Nucl. Phys. A. 122 (1968) 633.

[24] R.A.I. Bell, et al., Nucl. Phys. A. 133 (1969) 337. 